http://dx.doi.org/10.18359/ravi.1760

\title{
La poiesis en la facilitación del aprendizaje para el uso de las TIC en Educación Superior ${ }^{1}$
}

\author{
José Eduardo Padilla Beltrán ${ }^{2}$, Diego Armando Rincón Caballero ${ }^{3}$ \& José Arturo Lagos Sandoval ${ }^{4}$
}

Universidad Militar Nueva Granada - Universidad Escuela Colombiana de Carreras Industriales

Recibido, abril 12 de 2016

Concepto evaluación, mayo 20 de 2016

Aceptado, junio 30 de 2016
Referencia: Padilla Beltrán, J.; Rincón Caballero, D. \& Lagos Sandoval, J. (2016). "La poiesis en la facilitación del aprendizaje para el uso de las TIC en Educación Superior". Revista Academia y Virtualidad, 9, (2), 138-155

\section{Resumen}

En la actualidad, las universidades y demás instituciones de Educación Superior han asumido el reto de integrar aspectos de las TIC para prestar atención al papel del estudiante como arquitecto de su propio aprendizaje. En ese sentido, la problemática de un modelo pedagógico coherente con estas prácticas emergentes, ha incentivado diversos estudios acerca de perspectivas pedagógicas flexibles que consideren roles y funciones de docentes y educandos para aproximarse al conocimiento de una forma significativa. Por ello, desde un enfoque cualitativo en relación con la teoría fundamentada, el manuscrito presenta una interpretación de algunas posturas y reflexiones sobre la necesidad de una pedagogía flexible, sustentada en la posibilidad de un uso de las tecnologías educativas al servicio de una facilitación del aprendizaje mediante elementos creativos, participativos, críticos y axiológicos - poiesis-. En conclusión, se observa la incidencia de roles y funciones del docente como aspectos importantes por abordar para desarrollar este

\footnotetext{
${ }^{1}$ Resultado del proyecto de investigación: "Formación pedagógica del docente para el uso de TIC en la educación superior" (DIS-1242). Grupo de investigación: Pedagogía y Didáctica en la Educación Superior (PYDES), Universidad Militar Nueva Granada.

${ }^{2}$ Licenciado con estudios principales en Matemáticas, Universidad Pedagógica Nacional; Especialista en Orientación Educativa, Universidad Manuel Beltrán; Magíster en Supervisión y Administración Educativa, Universidad Externado de Colombia; Magíster en Evaluación en Educación, Universidad Santo Tomás; Doctor of Education, Newport University; Posdoctor en Educación, Ciencias Sociales e Interculturalidad, Universidad Santo Tomás. Docente Asociado y Director Centro de Investigaciones, Facultad de Estudios a Distancia, Universidad Militar Nueva Granada. Líder Grupo de Investigación: Pedagogía y Didáctica en la Educación Superior (PYDES). eduardo.padilla@unimilitar.edu.co
}

${ }^{3}$ Licenciado Diseño Tecnológico, Universidad Pedagógica Nacional; Magíster en Educación, Universidad Pedagógica Nacional. Asistente de investigación del Grupo de Investigación: Pedagogía y Didáctica en la Educación Superior (PYDES) de Facultad de Estudios a Distancia en la Universidad Militar Nueva Granada. diego.rincon@unimilitar.edu.co

${ }^{4}$ Ingeniero Electrónico, Universidad Distrital Francisco José de Caldas; Magíster Docencia, Universidad de la Salle; Investigador y Líder Grupo TEIN-ECCI, Universidad Escuela Colombiana de Carreras Industriales. jlagoss@eccci.edu.co 
tipo de orientación pedagógica y la prioridad de incluir al alumno como epicentro de la triada universidad-sociedad-empresa.

Palabras clave: Educación Superior, facilitación del aprendizaje, pedagogía flexible, poiesis, TIC.

\title{
Facilitating ICTs learning by poiesis in higher education
}

\begin{abstract}
Today, colleges, universities and other higher education institutions have taken up the challenge to integrate issues related to ICTs in order to pay attention to the student's role as a builder of his own learning. In that regard, the issues of a pedagogical model consistent with these emerging practices has prompted several analysis about pedagogical perspectives to think following flexible roles and tasks by teachers and students to reach a meaningful knowledge. Therefore, from a qualitative approach related to grounded theory, this paper offers an interpretation about some points of view and reflections to the need of a flexible pedagogy based on the feasibility to use educational technologies to make easier learning through creative, participatory, critical and axiological issues (poiesis). In conclusion, we observe the roles and tasks by teachers involved to address issues in order to develop this type of educational guidance and a priority to include the student as the cornerstone of a triad, i.e. university-society-business.
\end{abstract}

Keywords: higher education, facilitating learning, flexible pedagogy, poiesis, ICTS.

\section{A poiesis na simplificação da aprendizagem para o uso das TIC na Educação Superior}

\section{Resumo}

NAtualmente, as universidades e outras instituições de Educação Superior têm assumido o desafio de integrar aspetos das TIC para atender o papel do estudante como arquiteto da sua própria aprendizagem. Nesta direção, a problemática de um modelo pedagógico coerente com essas práticas emergentes, tem promovido diversos estudos ao redor de perspectivas pedagógicas flexíveis que considerarem papeis e funções de docentes e estudantes para se aproximar ao conhecimento de jeito significante. Diante disso, desde uma abordagem qualitativa no tocante com a teoria fundamentada, o manuscrito apresenta uma interpretação de algumas posições e reflexões sobre a necessidade de uma pedagogia flexível, apoiada na possibilidade de um uso das tecnologias educativas apontando a uma simplificação da aprendizagem através de elementos criativos, participativos, críticos e axiológicos -poiesis-. Em conclusão, observa-se a incidência de papeis e funções do docente como aspetos para abordar a fim de desenvolver esse tipo de orientação pedagógica e a prioridade de incluir o aluno como epicentro da triada universidade, sociedade, empresa. 
Palavras chave: Educação Superior, simplificação da aprendizagem, pedagogia flexível, poiesis, TIC.

\section{Introducción}

Las Instituciones de Educación Superior [IES] presentan un modelo pedagógico que orienta la formación dada en relación con un proyecto educativo y un énfasis que la caracteriza de otras instituciones; sin embargo, las distintas perspectivas pedagógicas que se tratan a nivel de facultades, dan cuenta de una diversidad de estrategias y propuestas pedagógicas que van de la mano con ciertos objetivos particulares de cada programa de pregrado o posgrado; o, por el contrario, distan del proyecto pedagógico planteado a nivel general. Sumada a esta condición, la singularidad del estilo pedagógico de los docentes trae consigo algunas experiencias que se pueden considerar exitosas o distintivas, pero a la vez se ven distanciadas por falta de comunicación o exposición ante otros pares. En ese orden de ideas y de acuerdo con lo planteado por González, Padilla y Rincón (2012), el rol emergente del docente en la creación de entornos de aprendizaje más contextualizados, implica una relación de propuestas pedagógicas flexibles, en tanto su integración devele aspectos generales y específicos de una formación para el mundo de la vida. Así pues, el objetivo del manuscrito consiste en desarrollar una puesta reflexiva en torno a la posibilidad de una pedagogía flexible alejada de una etiqueta o estandarización coercitiva, esto a través de una implementación consciente y pedagógica de las tecnologías de la información y la comunicación (TIC), como mediaciones dispuestas para articular dichas propuestas pedagógicas con base en nuevos modelos tendientes a la facilitación del aprendizaje.

En cuanto al paradigma de la facilitación del aprendizaje, se ponen en consideración los aportes de Quintero (2008); Bruner (2000); Padilla, García y González (2001) y Bustos (2005), quienes en conjunto manifiestan la importancia de realizar cambios paulatinos en las IES; transformaciones que se configuren de modelos tradicionalistas a entornos de aprendizaje más colaborativos, autónomos y acordes con los estilos de aprendizaje de los educandos. A su vez, el estudio incide en la importancia de trabajar un componente poiético, mediante una propuesta pedagógica basada en la capacidad innovadora y creativa de los estudiantes y los docentes para el planteamiento de actividades, contenidos y evaluaciones que lleven al alumno de un estado de imitador a un sujeto conocedor crítico y reflexivo (Isaza, 2011). De esta manera, se exponen los argumentos de Brito (2010); Beltrán (1996) e Isaza (2011) para resaltar la pedagogía poiética derivada de la conceptualización y el fundamento filosófico desarrollado por Enrique Dussel sobre la poiesis y su distinción de lo práctico y lo teórico.

En relación con el acervo de estudios anteriormente expuestos, en la parte metodológica se hace uso de un enfoque cualitativo para el análisis e interpretación de documentos primarios a partir de la teoría fundamentada de Strauss y Corbin (2002), mediante el desarrollo de cuatro etapas asociadas a la recolección y consolidación de una base de datos, la selección de información acorde al objeto de estudio, la codificación y clasificación de datos con el software Atlas. ti, y la depuración de los hallazgos a través del método de comparación constante y el muestreo teórico. Consecuentemente, el artículo presenta, en su primera parte, una aproximación conceptual al paradigma de facilitación del aprendizaje y los roles por tener en cuenta por parte del docente y el alumno, además de las implicaciones del uso de las TIC para incentivar un aprendizaje autodirigido y colaborativo. En la etapa de resultados se hace alusión a la pedagogía flexible como espacio de integración de múltiples perspectivas 
investigativas y experiencias del docente con el fin de articular estrategias idóneas a la formación de los estudiantes. Seguidamente, hay una profundización en la pedagogía de la poiesis y su articulación con el uso de las TIC en beneficio de su potencial de ofrecer recursos multimedia, hipertextos y diferentes recursos en aras de un ambiente de aprendizaje significativo para los alumnos y los docentes.

Finalmente, en las conclusiones se hacen algunas salvedades sobre la necesidad de imbricar un componente pedagógico coherente con las necesidades de formación de los estudiantes, prestando atención a su participación activa, las experiencias previas que trae y su papel como colaborador en las implementación de recursos TIC en pro de facilitar su aprendizaje. De otro modo, se rescata el fundamento de las pedagogías asociadas de Jerome Bruner en relación con el papel del docente y el estudiante a fin de incentivar una capacidad creativa e innovadora a través de elementos de la poiesis, enmarcados en una posibilidad de conocer en el discurso de la alteridad y el respeto a las diferentes formas de aprender, lo cual será menester del docente por medio de sus roles de tutor, consejero y mentor en el contexto de la Educación Superior.

\section{Facilitación del aprendizaje}

Para empezar a desarrollar el concepto de facilitación de aprendizaje y su articulación a la Educación Superior, es menester acercarse al significado del verbo facilitar, cuya noción se basa en hacer posible algo, tratando de potencializarlo y discernirlo en aras de fortalecer un proceso o actividad específica. Igualmente, Quintero (2008) plantea el facilitar como una acción que fortalece de manera sinérgica y progresiva el aprendizaje de modo autodirigido, capar de coordinar, corregir y desarrollar de manera integral una forma de acercarse al aprendizaje por el acercamiento y orientación de un saber o conocimiento particular. Así pues, la acción facilitadora en el contexto del aprendizaje en Educación
Superior, podría traducirse en la implicación de diferentes niveles de pensamiento, caracterizados por procesos autónomos y colaborativos que realizan de manera formal e informal un aprendizaje mediado por objetivos y labores deliberadas en una estrategia tanto pedagógica como didáctica.

El facilitar incluye el desarrollo de ambientes de aprendizaje para dicho fin sinérgico, pues se necesitan espacios, tiempos y dinámicas con el propósito de incentivar actitudes que permitan, de cierto modo, guiar y comprender las diferentes formas de acceder al conocimiento, logrando estados de reflexión mucho más significativos que la simple sumatoria de ítems y resultados generados individualmente por el educando. De este modo, al trasladar esta acción, producto de su verbalización, el actor que posibilita -o facilitador- será aquel conocedor de los procesos y dinámicas idóneas para consolidar trabajos colaborativos y autónomos con base en planteamientos consecuentes a propiciar metodologías, tecnologías, organización de asesorías y mentorías, como un conjunto armonioso de elementos en pro de incentivar el aprendizaje significativo en los alumnos. Etimológicamente, la palabra facilitar en latín confiere un sentido de realizar una acción con el mayor grado de funcionalidad, por ende, Quintero (2008) el facilitador se constituye como "[...] una persona experta en procesos grupales, con idoneidad en comunicación, dinámica grupal, efectividad grupal, toma de decisiones, diseño de talleres e intervención en crisis" (p.3).

De acuerdo a lo anterior, la educación ha venido asociando esta visión de facilitadores, no sólo lo relacionado con la acción del docente, sino además con un cambio de actitudes por parte de los estudiantes para que ellos mismos se conviertan en facilitadores de su aprendizaje, así como el orientador y apoyo del aprendizaje de sus compañeros. Por ello, en la actualidad se habla de romper paradigmas, como aquellos marcos culturales dotados de herramientas epistemológicas, susceptibles a orientar las 
formas de conocer y articular dicho saber a comunidades que permitan su anclaje a través de la validación y la aceptación; por consiguiente, se habla de un paradigma de la facilitación del aprendizaje, que permite, a la luz de lo expuesto por Bruner (2000), la creación de significados y la construcción de realidades para comprender el entorno que les rodea y, consecuentemente, transformarlo de acuerdo al nivel de aprendizaje y la creación de conocimiento acordes a relaciones de orden social, cultural, político y económico, dispuestas para dicho fin educativo.

En ese sentido, el papel que desempeña lo pedagógico es sumamente importante, en tanto el paradigma educativo de la facilitación del aprendizaje se sustenta en una transformación de la pedagogía tradicional enmarcada en la enseñanza, a una cambiante forma de incluir al educando de cara activa al proceso pedagógico, en la cual el docente al igual que el estudiante desarrollan procesos de construcción de aprendizaje y enseñanza. Al respecto, Bruner (2000) habla de una tradición pedagógica que muestra al docente como figura trascendente ante unos estudiantes sin ninguna experiencia o conocimiento alguno, lo cual se ha venido reformulando en aulas de clase con mayor participación de los educandos, al comprender al maestro también como aprendiz, lo que determina que si bien éste lleva la batuta o muestra el camino por seguir, éste se puede ir reformando o direccionando por las acciones y contribuciones de los estudiantes. Sin duda, se trata de un legado de los estudios constructivistas y los referentes de la psicología cultural que implican el concepto de intersubjetividad para profundizarlo y hacerlo dialogar en función de las relaciones e interacciones de los docentes y estudiantes en el aula, logrando dejar atrás la omnisciencia del profesor y entablando una visión más equitativa de cómo se aprende.

Sumado a esta condición de cambio, Bustos (2005) añade al proceso de transición pedagógica el ingrediente de una transformación social tendiente a una sociedad de la información, contexto en el cual el modelo pedagógico cambia al conceder múltiples opciones, medios y códigos al estudiante para acceder a la información y hacerla partícipe en su vida cotidiana; por ende, el alumno se constituye en el centro del proceso de aprendizaje, siendo responsable y consciente de la importancia de conocer de manera significativa a la luz de una guía o incentivo que será dada por el maestro, quien ya no es la figura autoritaria, sino un agente de cambio orientado a apoyar el trabajo autodirigido y reflexivo del educando. Isaza (2011) apoya esta noción de cambio, toda vez la perspectiva pedagógica converja en una relación menos vertical de transmisión y permute hacia una orientación más horizontal, cuya característica sea la de acompañar y guiar el aprendizaje del estudiante desde postulados pedagógicos concretos como los del constructivismo. De esta forma, las acciones del estudiante construyen una realidad en relación con el conjunto de experiencias y conocimientos previos utilizados para construir saberes encaminados a la misma construcción de docentes y estudiantes exploradores, actualizadores y ejecutores de un plan pedagógico sinérgico, capaz de dilucidar nuevas formas de aplicar lo aprendido a la resolución de problemas.

\section{Acciones $y$ recursos TIC usados por el docente para facilitar el aprendizaje}

Debido al impacto de las TIC en el campo educativo, más exactamente en el contexto de la Educación Superior, el docente acude a una serie de recursos y estrategias para lograr las condiciones de facilitación de aprendizaje del estudiante, teniendo en cuenta en principio su acción de moderador y regulador de dichos recursos para uso crítico, responsable y autónomo con base en los objetivos de aprendizaje. De esta manera, Barbera y Badia (2010) acentúan lo dicho en la acción docente como administrador de los recursos web y las plataformas virtuales, en tanto se creen unos principios de interacción y unas normas preestablecidas que permitan el diálogo proactivo y la comunicación tolerante 
entre los participantes, mientras los educandos proponen actividades, tiempos y modos para abordar dichas acciones de aprendizaje.

Entonces, el docente, en primera instancia, debe proporcionar a los educandos las herramientas conceptuales, tecnológicas y operativas para la búsqueda de información a través de diferentes recursos, bien sea físico o digital, pues durante el proceso de facilitación de aprendizaje será el estudiante quien busque la información y la retroalimente en función de sus intereses. Por ende, las habilidades de exploración son fundamentales para hallar información de calidad, acorde con las temáticas por desarrollar. El autoaprendizaje es una de tantas facultades afianzadas a medida que el estudiante sea capaz de buscar de modo adecuado la información necesaria de conocer algo en profundidad; sin embargo, también es útil en acciones como proyectos, trabajos colaborativos y construcción de objetos virtuales que implican de cierta manera una labor analítica, interpretativa y organizadora de la información consultada.

De otro lado, las TIC se caracterizan por ofrecer recursos multimedia diversificados que implican percepciones variadas, cuyo potencial se manifiesta en un aprendizaje más significativo; por consiguiente, la imagen se convierte en un recurso indispensable para facilitar el aprendizaje (Quintero, 2008), lo que posibilita un nivel más cercano a la intuición, la creatividad y la abstracción, producto de registros icónicos elaborados en relación con la experiencia perceptual y los códigos culturales para hacer las correspondientes lecturas. En esa medida, las imágenes puede equipararse a diagramas, fotografías, dibujos e ilustraciones asociadas a conceptos o referentes teóricos que pueden ir de la mano con el texto escrito o apoyos auditivos dispuestos conscientemente a fin de recrear segmentos de la historia o hechos específicos. Igualmente, la representación visual, producto de la memoria icónica, permite un ejercicio cognitivo activado por el hemisferio derecho con propiedades de síntesis, subjetividad y flexibilidad perceptiva.
Otro recurso valioso que se puede aplicar en términos de incentivar la facilitación del aprendizaje son las bases de datos, pues tal como afirman Barbera y Badia (2010) "[...] puede ser utilizada por el profesor para potenciar las habilidades de los estudiantes vinculadas con la búsqueda, clasificación e interrogación de datos, que pueden provenir de diferentes fuentes (como por ejemplo, de Internet)" (p.14). Al igual que las imágenes, puede ser una actividad complementaria a otras más globales, lo que implica procesos de gestión y administración de la información, muy útiles en trabajos colaborativos de tecnología e informática así como en proyectos de base telemática.

La instrucción en línea, por ejemplo, mediante guías o tutoriales se presenta como una fuente de información y recursos indispensable $\mathrm{y}$ articulada en forma enriquecida a las dinámicas de interacción entre estudiante y contenido en la web 2.0 o en los portales virtuales de aprendizaje. Dicha instrucción se lleva a cabo en el marco de una presentación de temáticas organizadas de acuerdo a objetivos, alcances y finalidades pedagógicas conocidas por el estudiantes para que estas temáticas puedan ser analizadas e interpretadas en una discusión o diálogos en los que se presenten diferentes puntos de vista sobre los temas abordados. De otro modo, la instrucción abre la puerta a otro recurso invaluable dentro del modelo de facilitación del aprendizaje, el cual se basa en la pregunta estratégica retomada por el docente en pro de estimular la participación activa de los estudiantes, pues con base en lo expuesto por Isaza (2011) representa “ [...] lo que está relacionado con la experiencia o puede ser representado en la imaginación, una pregunta que es útil o interesante responder o una situación que puede ser explicada y vale la pena explicar."(p.106), mientras que el docente debe profundizar a partir de observaciones que permitan en el mismo sentido al estudiante formular preguntas coherentes, que no sólo le permitan ahondar en el aula de clase sobre ciertos temas, sino además que sean útiles cuando pueda entrevistar o dialogar con 
un experto a través de diferentes técnicas de recolección de información usadas para indagar e investigar sobre un objeto de estudio en particular.

En cuanto a la formulación de preguntas o afirmaciones se propende por incentivar una dimensión comunicativa y expresiva del estudiante, tratando de generar discursos y dinámicas que permitan organizar ideas y comunicarlas desde puntos de vista argumentados y concretos. En el contexto de la educación virtual, en el cual la autonomía constituye un factor imperante, el docente es el promotor de preguntas orientadoras con base en las experiencias previas de los estudiantes y su capacidad de indagación, exploración y síntesis a la hora de poner sus argumentos en consideración en el escenario académico. Igualmente, en la educación virtual o presencial el ejercicio escritural se debe reforzar como herramienta indispensable para comunicar lo entendido, bien sea en el papel o en los nuevos formatos digitales como correos, foros y sesiones de chat como medios emergentes de comunicación sincrónica o asincrónica, donde el estudiante puede interactuar y dar a conocer lo que piensa de acuerdo a pautas de comunicación orientadas por el docente-tutor.

\section{El modelo de pedagogías asociadas de Jerome Bruner}

El concepto de pedagogía ha transmutado a lo largo de su preludio en diversas coyunturas y acontecimientos desarrollados en el transcurso de la construcción y declive de civilizaciones, mostrando particularidades a través de grandes pedagogos o escuelas enfocadas en resolver problemáticas de enseñanza-aprendizaje, pues en esas instancias, una educación favorable en los diversos círculos sociales se equipara a mejorar la calidad de vida y fomentar un crecimiento a nivel político y cultural. Así pues, los conceptos y perspectivas pedagógicas han variado en el transcurso de los años, a la par de cambios paradigmáticos y enfoques derivados de la psicología y las ciencias sociales. De igual manera, el concepto de educación se adopta para hablar de diversas circunstancias en las que se forma una persona o sociedad continuamente, es decir a lo largo de su existencia, pues tal como aclara Isaza (2011) "la educación se reconoce como un campo, cuyo marco social y cultural establece diferencias o similitudes por medio del debate y la confrontación".

Diversas posturas acerca del concepto de pedagogía sugieren su relación con la educación, más que disciplina o ciencia, como un saber del cual se desprende un conjunto de teorías, métodos y estrategias confinadas al estudio del proceso enseñanza-aprendizaje en un momento o referente social específico. En ese orden de ideas, el concepto de pedagogía se encuentra circunscrito a un marco de valores y acciones culturales que definen acciones y propósitos orientados al alcance de objetivos de aprendizaje, esto según Brito (2010) por medio de diferentes métodos y recursos. Por ello, y apelando a lo descrito por Isaza (2011), quien cita a Jerome Bruner, el referente pedagógico se pueden analizar desde tendencias o categorías, lo que implica la acción o el rol del estudiante, lo cual a su vez involucra una relación de diferentes modelos pedagógicos en torno a la actividad de aprendizaje del alumno. A continuación se describen, parafraseando a Isaza (2011):

$\checkmark$ El alumno como imitador: el objetivo pedagógico en esta categoría se basa en la adquisición de destrezas y habilidades que se adquieren e incentivan a través de la práctica.

$\square$ El alumno como aprendiz: la demostración o comprobación de lo que se aprende es producto de conocimiento avalado por una comunidad o sociedad académica, en tanto la enseñanza del conocimiento se da por medio de la cátedra y la repetición de ejercicios para el aprendizaje.

$\square$ El alumno como pensador: la discusión y el plano intersubjetivo permiten un aprendizaje colaborativo, cuyo afincamiento se encuentra en el uso creativo del conocimiento para la resolución de problemas.

$\square$ El alumno como conocedor: desde una perspectiva más crítica se pueden establecer discusiones argumentadas que permiten 
interactuar y conocer a profundidad el objeto de estudio.

\section{Método}

Teniendo en cuenta la complejidad del análisis de los datos o fuentes primarias, la investigación adopta un enfoque cualitativo (Sautu, et al., 2005; Valles, 2003), para establecer relaciones o tendencias categoriales provenientes del análisis de la revisión documental. Tal como refieren los autores citados, este abordaje permite obtener datos más consistentes $\mathrm{y}$, por consiguiente, una mayor multiplicidad de perspectivas sobre el estudio y sus analizadores. En este tipo de metodología, se consideran varias fuentes o documentos primarios acordes al objeto de estudio. A nivel de tratamiento de datos emergentes de la teoría general y sustantiva (Sautu et al., 2005), se recurrirá a la comparación constante (Valles, 2002) a partir de la teoría fundamentada, lo que confiere mayor fiabilidad y pertinencia de los resultados, por medio del análisis de datos con el software Atlas.ti.

Así las cosas, la teoría fundamentada crea conceptos y descriptores valiéndose directamente de los datos, provistos de teorías generales y sustantivas (Sautu, et al., 2005). De manera inductiva, la teoría se va consolidando a partir de un estudio emergente, en el cual no se hace uso de categorías generales aplicadas al objeto de estudio, sino que desde la realidad $\mathrm{y}$ un problema es que se van seleccionando y significando las categorías. Según Strauss y Corbin (2002), la teoría fundamentada se apoya en dos aspectos metódicos generales, a saber: (1) el método comparativo constante que codifica y analiza los datos de manera simultánea, el cual posibilita la creación de conceptos, i.e. "[...] Realizando una comparación continua de incidente con incidente, identifica sus propiedades, explora sus interrelaciones y logra integrarlo en una teoría que guarda una lógica razonable" (Giraldo, 2011, p 2); y (2) el muestreo teórico que luego de analizado en el fenómeno a profundidad, se exaltan nuevos elementos que puedan contribuir a ampliar los conceptos y la tendencia que se ha configurado. En relación con lo dicho, a continuación se muestran las etapas ejecutadas para la construcción conceptual del estudio.

Primera etapa: la búsqueda de los documentos se realizó a través de bases de datos virtuales especializadas como: ScienceDirect, SciELO, Redalyc, Dialnet, Google académico, entre otras. Además, Se contemplaron 60 documentos (artículos, libros derivados de investigación, memorias y tesis de posgrado) de los cuales 20 referían directamente al objeto de estudio: Docente y su rol en el uso de TIC en la Educación Superior (Ver Figura 1). Posteriormente, los documentos se recopilaron y guardaron como archivos digitales en los formatos PDF y RTF, cuyo fin es organizarlos y analizarlos bajo la herramienta o software Atlas.ti.

Segunda etapa: retomando la etapa 1 del proceso metódico, se prosigue con una depuración y clasificación de los documentos, por lo cual se hizo necesario establecer diálogos entre los integrantes del grupo para acordar un consenso de los documentos por incluir en el análisis, cuya necesidad subyace a partir de una información fiable y considerable para el estudio, puesto que en el análisis de datos cualitativos se busca la emergencia de información sobre el referente de una documentación enriquecedora y con diversos aportes para ser arbitrados en un marco teórico referencial, sucinto de interpretaciones y deducciones de carácter científico (lógico, verificable y sistematizable).

Terceraetapa:previoal procesodecodificación o clasificación de la información, se formularon dos categorías de tal manera que sirvieran como referente para la clasificación de la información. Estas categorías se construyeron desde la discusión y delimitación del tema con académicos e investigadores conocedores de perspectivas pedagógicas aunadas al uso de las TIC en Educación Superior, producto de investigaciones efectuadas en dicho campo 
educativo. Por ende, es muy importante el conocimiento exhaustivo que haya sobre el tema por parte del grupo de investigación. Las categorías de perspectiva pedagógica flexible y TIC y rol del docente en Educación Superior para el uso de las TIC, se convierten en los referentes de caracterización de las orientaciones pedagógicas asociadas desde la postura de Bruner (2000) más concurrentes y sus limitaciones con respecto al enfoque de la facilitación del aprendizaje como epicentro de transformación en las Instituciones de Educación Superior [IES].
Cuarta etapa: luego de codificar y establecer relaciones semánticas, se da paso a fusionar las unidades hermenéuticas -unir los archivos interpretativos de cada investigador- y así unificar los datos establecidos y usar un método de comparación constante (Strauss y Corbin, 2002) para realizar la reorganización de citas, códigos y memorandos -como elementos que ofrece el software para analizar los documentos y el corpus teórico-, a fin de esclarecer un análisis pertinente para el marco referencial que aborde la problemática del estudio y las relaciones conceptuales de manera inductiva a través del software Atlas.ti (véase figura 1).

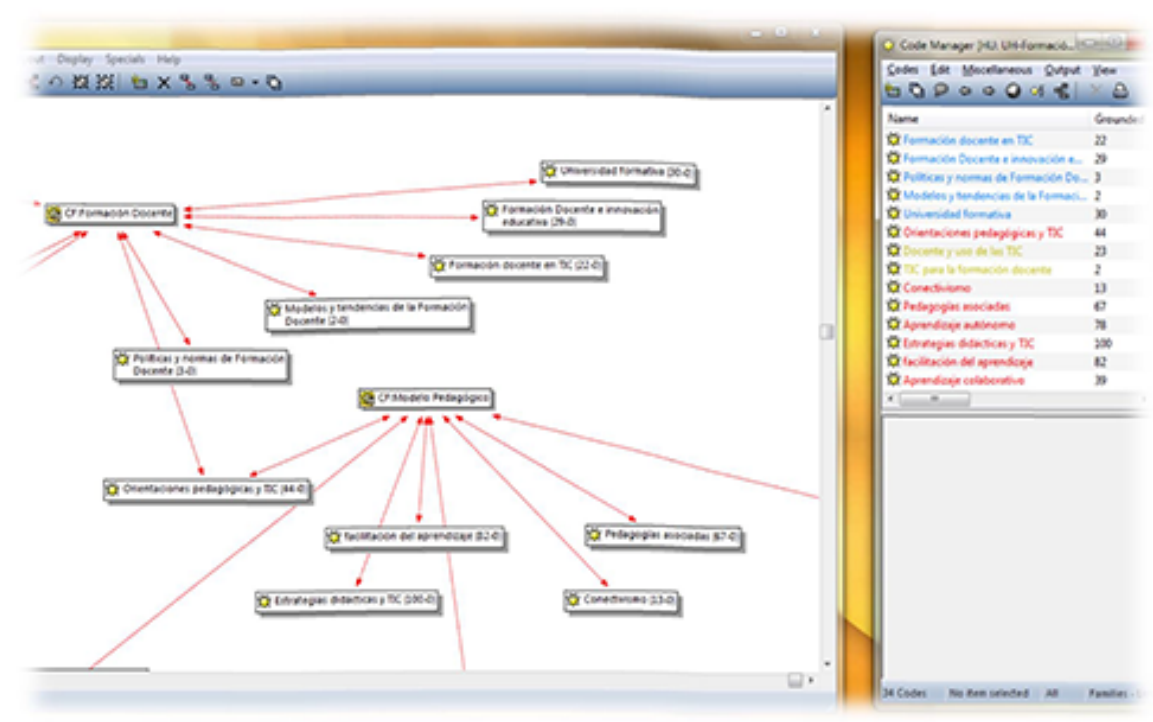

Figura 1. Fundamentación y red semántica de los códigos respectivos a las principales sub-categorias. Fuente: los autores.

En la primera fase de codificación, se aplicó una clasificación axial de los datos, de tal modo que permitiera reducir los documentos a unidades analizables, es decir, a fragmentos de texto con significado (citas), cuyo fin es organizar y dotar de sentido las lecturas efectuadas sobre los documentos primarios. La codificación axial permitió que los investigadores se familiarizaran con el lenguaje, la terminología y las subtemáticas de los documentos, lo que posteriormente serviría en la evaluación de las categorías aplicadas y crear nuevas categorías necesarias para describir con mayor detalle la información.

Como segunda fase en la codificación, se adicionó el método de codificación abierta para construir nuevas categorías, de tal manera que permitieran describir la información. Para este fin, se realizó la comparación de los códigos y sus respectivas citas, leyendo en profundidad $\mathrm{y}$, de ser necesario, ajustando la información en nuevos códigos y jerarquías, lo que posibilita una mayor comprensión y descripción de las categorías creadas en un principio (Valles, 2003). Resultados

Los resultados están encaminados a mostrar la relevancia de una pedagogía poiética que 
trasciende más allá de su uso en las facultades o programas de arte, diseño y filosofía, para prestar atención a su nivel propositivo, creativo e innovador desde la producción con sentido en aras de vincular a los educandos a un proceso reflexivo en el marco de la facilitación del aprendizaje en la Educación Superior. Para ello, el manuscrito muestra la relación del rol del docente (aunque el rol del estudiante es más importante en este paradigma, en el estudio se enfoca la necesidad de reevaluar las acciones del docente), con la transformación de prácticas imitadoras a un conocimiento contextualizado, producto de vincular estrategias pedagógicas flexibles y acordes a ritmos de aprendizaje diversificados, pero no inconexos de las actividades, contenidos y evaluaciones que desarrolla el docente en conjunto a los aportes colaborativos y autónomos del educando. A la luz de lo expuesto, el siguiente esquema (véase figura 2) pretende representar con mayor claridad lo anteriormente dicho, lo cual se desarrollará a posteriori de acuerdo a los hallazgos interpretados en los datos registrados y organizados en las unidades hermenéuticas.

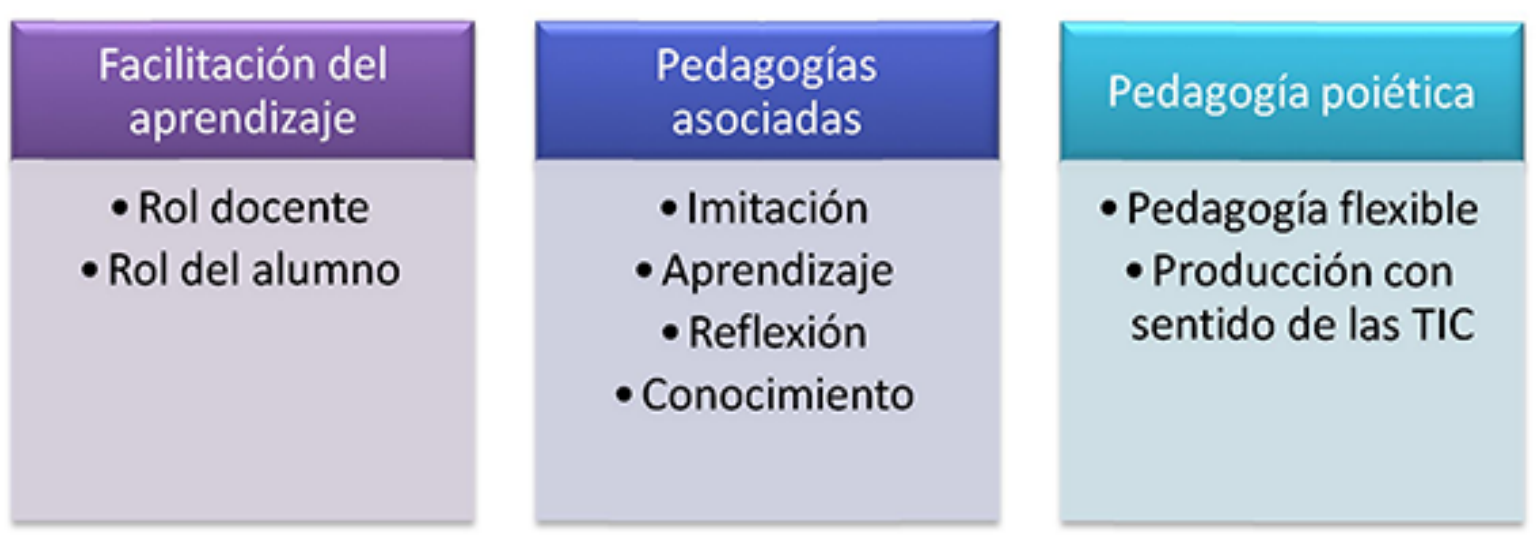

Figura 2. Pedagogia poiética en relación con una facilitación del aprendizaje. Fuente: los autores.

\section{Hacia una pedagogía flexible}

De acuerdo a lo expuesto en el anterior apartado, la pedagogía -en tanto discurso o gran relato- debe conservar una relación íntima con lo cultural, puesto que en medio de su correspondencia con el campo educativo puede contribuir a transformaciones sociales, como también puede entrar en detrimento con las políticas y normas educativas planteadas para un desarrollo social. En relación con estos presupuestos, las políticas de autonomía institucional y las reformas políticas con respecto a los modelos de educación, se vuelcan hacia una perspectiva flexible, una flexibilidad que no se puede confundir con libre albedrío o improvisación sobre la marcha de una perspectiva educativa, puesto que la flexibilidad -entendida a la luz de De Benito y Salinas (2005)- puede configurar la necesidad a nivel de educación presencial, virtual o mixta de una orientación dialógica en la cual existan debates, especulaciones y participación de la comunidad educativa para la toma de decisiones sobre el rumbo de la educación; por consiguiente, el factor de flexibilidad está asociado a lo comunicativo, lo intercultural y las renovaciones a nivel administrativo, pedagógico y didáctico con fines de mejorar la calidad en los diversos grados de formación. 
En acopio a esta flexibilidad, se puede recurrir a la analogía de la Suite planteada por Alís (2005) quien referencia una danza antigua que emplea varias corrientes, lo cual sucede con lo pedagógico, pues de forma armoniosa usa diversos movimientos de corte pedagógico, didáctico y estratégico para lograr desde una perspectiva histórico-social desarrollar un entramado de relaciones con el fin de alcanzar los objetivos de aprendizaje del momento. Inclusive se puede extender la analogía de la Suite a sus significaciones de hospedaje y ofimática como una serie de instancias y aplicaciones que dan cuenta de un propósito asociado a lo pedagógico desde la multiplicidad de conceptos, recursos y estrategias utilizadas para atender el propósito de educar de manera consciente y apropiada a una sociedad bajo diversas formas.

La pedagogía encauzada a lo flexible lucha en contra de una pedagogía estándar (Isaza, 2011), que usa la dicotomía entre lo bueno y lo malo, lo que se debe y no se debe, el uso indiscriminado de una idea al punto de volverla ideología, de usar la razón para el racionalismo exacerbado y la lógica de un saber para convertirla en patrón de secuencias por replicar. Adicionalmente, Isaza (2011) habla de la pedagogía estándar, así:

[...] En la pedagogía estándar, el desenlace suele ser una actitud de fracaso y decaimiento de la autoestima, para los estudiantes de más bajo logro, y al mismo tiempo de monotonía, mínimo esfuerzo y desencanto para los estudiantes de logro más alto. (p.122).

En contraposición a esta pedagogía estándar, la pedagogía flexible dinamiza el uso de diferentes perspectivas pedagógicas, a través de planteamientos de reconocimiento de la diferencia, de la inclusión de aspectos y ritmos de aprendizajes diversos y abogando por múltiples escenarios para el desarrollo de dinámicas variadas que favorezcan la actividad de participación e interacción, bien sea con el contenido, los compañeros o el ambiente. En efecto, la flexibilidad compromete mayor esfuerzo en el proceso pedagógico, puesto que lleva a docentes y estudiantes a modificar su quehacer e incentivar practicas emergentes para alcanzar objetivos de aprendizaje de un orden significativo que no sólo se asocien a lo cognitivo, sino además a lo actitudinal.

Especialmente, en la pedagogía no estándar prevalecen los encuentros entre reflexión e investigación (Freire, 2004), pues como se ha venido manifestando, sin una modificación paulatina de las actitudes y las estrategias de enseñanza-aprendizaje, se puede caer en el error de fomentar retos y propuestas obsoletas, aún más en la actual sociedad de la información y con el referente de la complejidad, la constante formación que implica una renovación encarnada en el conocimiento de nuevos espacios, actividades y orientaciones pedagógicas dedicadas a recrear y diseñar desafíos significativos y de interés para los actores educativos, reconociendo en el educando un papel activo y capaz de aportar al desarrollo de dichas máximas pedagógicas.

\section{La pedagogía de la poiesis}

Antes de empezar a esclarecer las relación poiética y pedagógica, se hace necesario considerar una aproximación conceptual de la poiesis, estableciendo en principio diferencias con la práctica, pues en diversas literaturas se resuelve por homologarlas sin distinción de su connotación. De ahí que Beltrán (1996) distinga tres tipos de inteligencia derivados de los preceptos griegos para describir las funciones del ser humano: lo práctico, lo poiético y lo teórico. Evidentemente, cada una contribuye al desarrollo del sujeto social, y expresa unas relaciones lógicas para dar cuenta de la inteligencia colectiva del ser. Asimismo, Beltrán (1996) recurre a la técnica y a la poiesis para referirse a las primeras civilizaciones en relación con las facultades de cooperación para la subsistencia y la búsqueda de recursos, logrando así modificar lo natural para adaptarlo y captar en esencia para luego implementarlo con diversos propósitos. 
El conocimiento del mundo y del entorno lleva al hombre a establecer rumbos y abrirse a posibilidades inimaginables, pues sistemas abiertos como el cultural moldean no sólo su forma de actuar sino también la cultura donde se desenvuelve; por ende, el hombre busca en principio con la técnica reformar $\mathrm{y}$ acondicionar para luego otorgar sentido y relación de productividad y existencia a esas cosas o elementos a través de la poiesis. Por su parte, Enrique Dussel (citado por Beltrán, 1996) se refiere a la inteligencia poiética como "[...] un a priori de la inteligencia teórica. La instancia productiva condicionará materialmente toda instancia especulativa, ideológica y aún científica (si se entiende la ciencia como una teoría metódica)" (p. 5).

Lo planteado por el autor da a entender un preámbulo de diferencia entre lo teórico y lo práctico mediado por el recurso poiético, pues el hombre no se acerca de incipiente mano a su esencia y la visión de mundo para comprenderlo y explicarlo, ya que en primera medida lo habita, se adapta y suple sus necesidades por medio de la transformación del entorno a conveniencia de su subsistencia.

En ese orden de ideas, su acercamiento a lo teórico se produce en la medida en que su necesidad sea suplida bajo el conocimiento del cosmos y el lugar que habita, para luego entrar en él con mesura y a profundidad como un referente de posibilidades y cambios que se van dando en medio de lo tangible e intangible de la situación adaptativa. A lo largo de la historia, el hombre ha modificado e incentivado sistemas y organizaciones, lo que le permite en un plano cultural conocerse y representarse, y de la misma forma dando cabida a un conjunto de símbolos, arquitecturas e instrumentos que posibilitan ese conocimiento, cuya aplicación inauguró materiales e instrumentos que le permitieran producir bajo una instancia poiética; es decir, el fin y posibilidad de articularlos a una función, a lo que Aristóteles ha descrito en la metafísica como una facultad “dianoética" (Beltrán; 1996: 5).
Entonces, se puede ir exhortando la poiesis como una actividad productora que implica o no un referente técnico concurrente, pues puede haber una racionalidad en la actividad técnica; sin embargo, desde la poiesis se encuentran presentes la racionalidad y la reflexión de la producción en medio de aspectos metódicos. Por el contrario, la técnica se convierte en un proceso con ciertas máximas de habitualidad, experiencia y descubrimientos conducentes a la creación de algo por medio del análisis de sus partes, más aún la poiesis como actividad productora es conducente a un discurso que propende por lo teórico para el conocimiento y ajuste de los elementos técnicos. En otras palabras, Isaza (2011, p.142) plantean las vicisitudes de cada una de éstas a continuación:

- Techné: saber-hacer técnico que demanda destrezas y habilidades.

- Praxis: campo estructurado y avalado por un consenso de especialistas de conocimientos organizado en problemas, orientados a soluciones concretas con objetivos predeterminados.

- Teoría: conjunto de reglas y principios conceptuales que suponen valores universales y posibles aplicaciones prácticas.

- Poiesis: hacer transformador-creador que configura procesos, genera productos, y construye conocimiento y sentido.

De lo expuesto, se puede resaltar la condición inventiva o creadora de la poiesis, capaz de establecer un conocimiento emergente en el marco de lo teórico y lo práctico. Claro está que no es menester de la poiesis indagar sobre lo ya configurado en el acervo teórico, ni tampoco lo inherente del proceso o aplicación discernido de lo práctico o en el deber ser que se retoma desde lo ético y lo político en el obrar del sujeto, se trata de una poiesis del hacer una producción exógena, libre de lo superfluo o las condiciones de su creación (Beltrán, 1996). En esa medida, la poiesis no es ajena a la racionalidad, por lo cual dista de un empirismo al tener claridad en sus procesos $\mathrm{y}$ fundamentos, siendo propios y producto de una reflexión de lo que se construye $\mathrm{y}$ 
produce. Beltrán (1996), al respecto, afirma sobre la base de los supuestos de Tomás de Aquino de la poiesis como un fundamento importante en el arte, en cuanto se da una racionalidad pertinente para dar finalidad a una producción elocuente. En resumidas cuentas, la poiesis puede cruzar, mas no rebasar, los límites de lo práctico y lo teórico a partir de la trascendencia de obstáculos que se pueden dar desde un campo meramente intelectual $u$ otro simplemente empírico.

Recapitulando, la teoría es algo abstracto con referencia al ámbito del ser para el estudio de una serie de principios y leyes, mientras que la práctica acude a la técnica en el orden del bien común (Beltrán, 1996), y la poiesis está enmarcada en los preceptos de lo estético por medio del orden y la coherencia del producto emergente del conjunto de técnicas o procesos designados para una racionalidad que puede implicar a la pedagogía desde lo sugerido por Beltrán (1996), es decir, “[...] asumida dentro de la concepción de la alteridad, por ende de la racionalidad dialógica participativa-, la pedagogía y la poiesis [...]". En dicho marco reflexivo sobre la poiesis, se puede vislumbrar un epicentro pedagógico que trasciende de lo instrumental y devela un nivel de expresión característico en la dicotomía de la libertad de expresión y los lineamientos de una didáctica acoplada a las metodologías de una disciplina (Isaza, 2011).

Precisamente del concepto puesto a consideración sobre la poiesis, se desprende una manera de acercarse a una pedagogía de la poiesis, en tanto difiere de una postura equiparada a la técnica, tal como asiente Habermas (según Isaza, 2011), en el sentido en que se revalúa el hecho de confinarla a la manualidad y al facto propiamente, pues detrás de ella se encuentra configurado el ámbito y los medios utilizados por el hombre para comprender la práctica. Por consiguiente, la praxis pedagógica se reconoce en las acciones $\mathrm{y}$ medios destinados a resolver problemas en el marco de cierta disciplina, a través de elementos de enseñanza-aprendizaje puntuales a objetivos previamente establecidos. En la praxis pedagógica y de acuerdo a la disciplina, las ideas subordinan la acción práctica, pero de otro lado y específicamente en las artes, la experiencia y el carácter de otorgar sentido, van dando cuenta de principios por articular de lo singular a lo universal, pues a medida que se hace práctica puede revalidar diversos aspectos teóricos.

Entre lo teórico y lo práctico, entonces, se mueve lo metodológico, validando la acción de tesis e hipótesis en función de unos métodos que permitan una verificación de lo práctico en sistemas referenciales teóricos y viceversa. Lo anterior se pone a consideración, pues en la poiesis ambas tienen un valor equiparable al hecho de una función creadora que orienta los principios teóricos, la praxis y la práctica, considerando la praxis y la teoría, no sólo en el acervo de conceptos, sino además como una guía de experiencias para el desarrollo de nuevas técnicas. Así pues, la pedagogía poiética favorece el paradigma de facilitación del aprendizaje, entablando una relación desde el paradigma constructivista sobre la base metacognitiva y la articulación de metodologías alternas para la comprensión y el desarrollo de habilidades y actitudes productivas y reflexivas. Igualmente, el descubrimiento es una parte importante en la pedagogía poiética, en la medida en que ayuda a los sujetos a contextualizar el aprendizaje a partir de lo práctico para ir develando elementos teóricos, ambas de carácter relevante para el aprendizaje significativo y la retroalimentación, producto de la orientación de las indagaciones realizadas por interés propio.

Con el mismo propósito, la pedagogía poiética asume el valor de la autonomía como una aproximación contundente para aprender a aprender (Isaza, 2011), cuyo marco de acción se mueve en la interpretación de los hechos y el aprendizaje en función de un pensamiento volcado en el hacer. De esta forma, la pedagogía poiética, afirma Isaza (2011), "[que] es igualmente importante 'aprender a 
desaprender' para poder descubrir e reinventar, pues sin ruptura y quiebre de paradigmas, modelos y reglas no hay emergencia de nuevas posibilidades y no tiene lugar el proceso de creación" (p.146). Por ello, la creatividad se asume como esa oportunidad de romper dichos paradigmas y establecer nuevos horizontes hacia una experiencia que permita conocer y determinar niveles teóricos para explicar los resultados y el proceso desarrollado en la producción de experiencia.

En suma, la poiesis implica un hacer y conocer de manera creativa y trascendente, en la medida en que se reconfiguran procesos, escenarios y productos de conocimiento con un alto grado de significancia. Siendo así, la creación es subsidiaria de la poiesis toda vez se presenten aspectos de interés y motivación que impliquen dedicación para derribar obstáculos y creer en lo que se está haciendo. En otras palabras "es un devenir de los modos de hacer que se despliegan en medio de las tensiones entre el deseo, la necesidad, lo conocido, la incertidumbre y la indeterminación consubstanciales a los procesos de creación" (Isaza; 2011:150). De ahí que la técnica sea reflexionada, la praxis contextualizada y la decantación teórica a partir de las revisiones conceptuales sean elementos provistos como una forma de acercarse a dicha pedagogía poiética.

Así las cosas, la pedagogía poiética también indaga por una intersubjetividad, tanto del docente y estudiantes como toda la comunidad educativa (Isaza, 2011), pues todos aprenden y enseñan en la medida de contribuir a los procesos pedagógicos y didácticos en cuestión. El docente como orientador y guía en la facilitación del aprendizaje, comprende que su quehacer debe ir de la mano de su asertividad poiética; la creatividad en sus clases y la implicación de diversas estrategias didácticas, producto de su rol de investigador, hacen que la interacción y la actividad creadora del estudiante sea más enriquecedora a la hora de adaptar procesos de técnica, praxis y teoría a las especificidades y experiencias de cada alumno.
Se puede pensar que la poiesis se aparta de las tres condiciones mencionadas; sin embargo, y como afirma Isaza (2011), pretende renovarlas desde una transformación contextualizada a través de la crítica y la vinculación de niveles de conocimiento que van de lo sensitivo, lo perceptivo, el conocimiento y la metacognición para el empleo de metalenguajes y recursos de expresión enmarcados en lo icónico, simbólico, narrativo y lo no verbal como otras alternativas de aproximarse al saber ser y hacer.

Para concluir, las dimensiones y características de una pedagogía poiética recogen elementos de lo reflexivo, lo comprensivo y dinámico de acuerdo a marcos teóricos, praxeológicos y técnicos distintivos en cada disciplina, integrando aspectos de la lógica, del lenguaje y de la expresión artística acorde al desarrollo de la percepción, la razón, la memoria, la intuición, la creatividad, la imaginación y la exploración como recursos indispensables en un ambiente de aprendizaje susceptible de potenciar la metacognición y emplear las experiencias y el papel activo del educando en pro de una reestructuración de su aprendizaje tendiente a lo crítico, autónomo y participativo.

\section{Enfoque poiético y flexible en la educación sustentada en TIC}

Una educación cada vez más cambiante en el contexto de la sociedad de la información, prevé el uso de las tecnologías de la información y la comunicación [TIC] como una base de formación con capacidades de establecer marcos flexibles, creativos y autónomos en la educación. En esa medida, la relación pedagógica y tecnológica, aunque pudiese entrar en conflicto, también consigue aunar un puente educativo caracterizado por incluir al educando de manera activa y propositiva en el trayecto de su formación. Es así como las tecnologías y los ambientes de aprendizaje dotados de espacios virtuales se muestran como un apoyo constante por implementar en la solución de particularidades de un aprendizaje continuo y basado en las 
expectativas de los alumnos. De igual forma, los contextos laborales, también modificados por la inscripción de diversas mediaciones tecnológicas, requieren personas proactivas, siempre tendientes a formarse y a adquirir habilidades en el campo de la comunicación, la interacción y el manejo de medios tecnológicos con el propósito de realizar un trabajo acorde a las demandas complejas del momento; por ende, la informática según Brito (2010) y los recursos inteligibles del discurso tecnológicos se vuelven imprescindibles a la hora de fomentar un aprendizaje significativo, coherente con lo expuesto anteriormente sobre el aprendizaje colaborativo y la capacidad autónoma desde la poiesis de dinamizar producciones con sentido y valor a nivel sociocultural.

De acuerdo con lo enunciado por Brito (2010), las teorías constructivistas se presentan como un referente por articular en los espacios virtuales o mixtos de aprendizaje, en la medida en que se van generando de manera sinérgica modelos y estrategias de aprendizaje sustentados en tecnología colaborativa, como en el caso de la web 2.0, pues a través de dichas mediaciones tecnológicas lo que se busca es un conocimiento pertinente del contexto, mediante las cuales se aborda la experiencia y conocimientos previos del estudiante en función de una recreación y construcción de problemas que sirvan de pretexto para aplicar conceptos y teorías orientadas por los docentes en un área disciplinar. A la luz de esta conjetura, lo importante en la conexión de lo pedagógico y lo tecnológico se circunscribe en la preponderancia de unos objetivos de aprendizaje y en una evaluación retroalimentada con mayor índice formativo que sumativo, dando cuenta de los procesos implicados por el estudiante para conocer y alcanzar dicho objetivo, tanto de manera individual como a nivel colaborativo.

La triada de este modelo de aprendizaje se puede comprender a través de tres elementos; el primero, la teoría de aprendizaje, inherente a la vinculación de referentes teóricos a nivel pedagógico y didáctico sobre la facilitación del aprendizaje por medio de una significación, contextualización y flexibilidad cognitiva (Brito, 2010); en segunda instancia se encuentra el modelo relacional (Brito, 2010), que cuenta con una serie de etapas concomitantes con el trabajo individual, colaborativo o tutorial del docente en acopio a las formas de tratar diversas temáticas según la complejidad y; las estrategias didácticas, como tercer pilar que determina-de acuerdo a la teoría de aprendizaje- ¿cuáles ejes, etapas y recursos son los más adecuados para el desarrollo de un aprendizaje significativo?. Consecuentemente, el modelo de aprendizaje se debe valorar de manera interna por docentes y educandos, y de forma externa, por directivos y pares, de tal forma que puedan lograr ajustes o regularidades del enfoque pedagógico, involucrando la participación, orientación y motivación del estudiante para ser protagonista y contribuyente a un aprendizaje encaminado a sus intereses y expectativas.

De otro lado, esta triada está en la obligación de adoptar enfoques flexibles, toda vez sea coherente el análisis de las diferencias de los ritmos de aprendizaje, cuya reflexión debe dar cuenta de los niveles de argumentación, creatividad y comunicación que manejan cada uno de los educandos y así integrar grupos formales, o de base, homogéneos o heterogéneos, según el caso, en aras de fortalecer e incentivar otras habilidades a través de la interacción con sus compañeros en la construcción de aprendizaje relevante para diversas esferas, tales como lo social, lo cultural e inclusive en el cuidado y aprecio de su entorno a nivel ecológico (Isaza, 2011). Por ello, el constructivismo y el referente social de esta corriente pedagógica se muestran como una alternativa por tener en cuenta en la relación pedagogía-tecnología mediante estrategias conducentes a una dialógica entre sujetos, medios y acciones cohesionadas a los factores de trabajo colaborativo y discursos solventados de argumentación poiética para la reflexión teórica, propios de un panorama en el cual el estudiante puede enseñar al maestro 
y a sus compañeros, sin demeritar el hecho de tener respeto por las opiniones de los demás y logar acuerdos racionales en palabras de Isaza (2011), producto de la indagación y la exploración que ofrecen los nuevos escenarios propuestos en la mediación tecnológica en el campo educativo.

Para finalizar, no basta con recursos y soportes tecnológicos de última generación, cuyo valor real en el campo educativo consiste en ofrecer mediaciones para la construcción de ambientes de aprendizaje idóneos; por consiguiente, y superando la visión de los ambientes de aprendizaje como espacios físicos acondicionados, los ambientes como climas llenos de propósitos, metas y expectativas se fundamentan en verdadera magnitud con funciones pedagógicas encaminadas a crear situaciones y acciones pertinentes para la facilitación del aprendizaje, en la medida en que lo didáctico contribuya a dichos ambientes en la implicación de métodos y estrategias que vayan de la mano con esa perspectiva pedagógica, planteada en este documento como no estándar; flexible en sus dimensiones de interacción y poiética cuando se incentiva la producción creativa y racional en relación con una praxis significativa.

\section{Conclusiones}

Los procesos de facilitación del aprendizaje se incorporan en la Educación Superior para romper paradigmas centrados en la enseñanza, lo que muestra paulatinamente las bondades de un aprendizaje centrado en los intereses y particularidades cognitivas y actitudinales de los estudiantes. No obstante, dicha acepción no debe crear ruido en el sentido de demeritar la dinámica de la cátedra y la importancia de las interacciones presenciales, pues se trata más de una gradualidad en la cual se haga conciencia de la emergencia de nuevos roles y funciones, tanto de docentes como de estudiantes, para abordar la complejidad de un aprendizaje para la vida. De este modo, la tecnología aplicada a la educación provee una reflexión constante que permita comprender la iniciativa gradual de la colaboración, la participación activa y la disposición como factores claves con el fin de vincular contenidos, actividades y evaluaciones pertinentes en estos espacios o aulas de aprendizaje que usan las TIC.

La teoría fundamentada por medio de la codificación de documentos primarios, muestra que la categoría perspectiva pedagógica flexible y TIC a través de subcategorías de orientaciones pedagógicas, pedagogías asociadas y facilitación del aprendizaje, posee una alta fundamentación, producto de estudios e investigaciones que tratan de develar la importancia de una pedagogía que no sesgue y demerite las habilidades de los docentes y estudiantes, en tanto se logre armoniosamente la integración de diversas perspectivas pedagógicas y didácticas al servicio de un reconocimiento del contexto, la historia y los énfasis formativos que dan las IES para propiciar una educación de calidad y pertinente a fin de resolver problemáticas del entorno. En esa medida, la pedagogía de la poiesis se presenta como una alternativa centrada en incentivar la producción creativa, diferenciada ésta de la práctica y la teoría, recurre a ellas para reflexionarlas, adaptarlas o modificarlas metodológicamente, en relación con unos elementos estéticos, axiológicos y culturales que dan cabida a pensar el otro alteridad- en las acciones que se realizan; es decir, el quehacer profesional incide en el alumno, tanto como las acciones del educando llevan a que el docente reflexione sobre su intervención pedagógica.

De lo anterior, no se puede esperar que una facilitación del aprendizaje vaya de la mano con modelos pedagógicos coherentes y flexibles, sin reevaluar el rol del docente y los recursos que utiliza para generar un estado de crítica y reflexión constante acerca de su orientación profesional y el impacto que tienen en la comunidad académica que lo acoge. Por consiguiente, la transformación gradual de una labor docente para asumir aspectos de la pedagogía poiética, se imbrica con funciones enmarcadas en la tutoría, la consejería y la 
mentoría, en calidad de niveles de interacción con el educando que lo acercan más a una visión guiada de la institución educativa y el mundo externo, pues al ver la universidad ya no como claustro, la triada universidad, sociedad y empresa conllevan a un aprendizaje más acorde a las necesidades del entorno, y en esa medida el docente está en capacidad de guiar, orientar y motivar las acciones autodirigidas del educando para desempeñar un quehacer idóneo en lo personal y en lo profesional.

\section{Referencias}

Alís, M. (2005). El uso de las TIC y de los materiales didácticos de carácter interdisciplinar y multimedia en el área de música. Una reflexión a partir de la propia experiencia docente. En: M. Casanovas, M.C. Jové y A. Tolmos (Eds.). Las TIC en la formación del profesorado, las perspectivas de las didácticas específicas (pp. 41-54). Lérida: Universidad de Lleida.

Barbera, E. y Badia, A. (2010). Hacia el aula virtual: actividades de enseñanza y aprendizaje en la red. En: Revista Iberoamericana de Educación, 1-21.

Beltrán, F. (1996). Praxis y poiesis en la pedagogía y la didáctica. En: Lúdica Pedagógica, 1(2), 11-16.

Brito, P.R. (2010). Propuesta de una guía didáctica, para aplicar la técnica b-Learning, bajo la plataforma Moodle, que optimice los procesos de enseñanza-aprendizaje de las diferentes asignaturas de la carrera de Ingeniería en Informática y Multimedia de la Universidad Internacional del Ecuador. En: Revista Generación Digital, 9(1), 35-39.

Bruner, J. (2000). La Educación, puesta a la cultura ( $3^{\mathrm{a}} \mathrm{Ed}$.).Madrid: VISOR.

Bustos, A. (2005). Estrategias didácticas para el uso de TICs en la docencia universitaria presencial. Barcelona: Pontificia Universidad Católica de Valparaíso.
De Benito, B. y Salinas, J. (2005). Situaciones didácticas en los entornos virtuales de enseñanza-aprendizaje (EVEA) en la enseñanza superior: elaboración de un instrumento de análisis. Presentado en la Conferencia EDUTEC 05. Formación del profesorado y Nuevas Tecnologías. Recuperado de gte.uib.es/pape/gte/ publicaciones/author/1011

Freire, P. (2004). Pedagogía de la autonomía. Sao Paulo: Paz e Terra S.A.

Giraldo, M. (2011). Abordaje de la Investigación Cualitativa a través de la Teoría Fundamentada en los Datos. En: Actualidad y Nuevas Tendencias. 2 (6).

González, K., Padilla, J.E. y Rincón, D. (2012). El docente en contextos b-Learning. Bogotá: Universidad Militar Nueva Granada.

Isaza, J.F. (2011). Modelo pedagógico de la Universidad Jorge Tadeo Lozano. Recuperado de http://avata.utadeo.edu.co/documentos/ pdf/modelo_pedagogico_web.pdf

Padilla, J.E., García, L. y González, M. (2012). Fundamentos en pedagogías contemporáneas para la educación a distancia y virtual. Bogotá: Universidad Militar Nueva Granada.

Quintero, G. (2008). Facilitación de procesos de aprendizaje en PSE. Programa regional de formación de facilitadores en metodologías innovadoras de planificación, seguimiento y evaluación para el desarrollo rural (PREVAL). Recuperado de http://www.asocam.org/portal/ sites/default/files/publicaciones/archivos/ Elementos_basicos_facilitacion.pdf

Sautu, R., Boniolo, P., Dalle, P. y Elbert, R. (2005). Manual de metodología. Buenos Aires: Colección Campus Virtual.

Strauss, A. y Corbin, J. (2002). Bases de la investigación cualitativa. Técnicas y procedimientos para desarrollar la teoría fundamentada. Colombia: Editorial 
Universidad de Antioquia.

Valles, M. (2002). Ventajas y desafíos del uso de programas informáticos (e.g. ATLAS. ti y MAXqda) en el análisis cualitativo. Una reflexión metodológica desde la grounded theory y el contexto de la investigación social española. Documento de trabajo Serie Sociológica (2001). Fundación de estudios Andaluces.

. (2003). Técnicas cualitativas de investigación social reflexión metodológica y práctica profesional. Madrid: Editorial síntesis. 